\title{
Primary care provider perspectives on using genomic sequencing in the care of healthy children
}

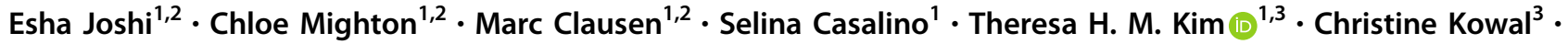 \\ Catherine Birken $^{2,3} \cdot$ Jonathon L. Maguire ${ }^{1,2,3} \cdot$ Yvonne Bombard $^{1,2}$
}

Received: 19 April 2019 / Revised: 3 October 2019 / Accepted: 29 October 2019 / Published online: 5 December 2019

(c) The Author(s), under exclusive licence to European Society of Human Genetics 2019

\begin{abstract}
Genome sequencing (GS) studies involving healthy children can advance scientific knowledge of genetic variation. Little research has examined primary care providers' views on using GS in this context. This study explored primary care provider perspectives on the use of GS in research and the care of healthy children. We conducted semi-structured interviews with 16 providers discussing their views on GS research and receiving results. Interviews were analyzed by thematic analysis and constant comparison. Participants were family physicians (11/16) and primary care pediatricians (5/16) in practice for $>10$ years (11/16). Participants valued GS in healthy children for research purposes; however, opinions diverged on using the results in primary care. Proponents valued using results for surveillance and prevention in healthy children. Skeptics questioned the clinical utility of results and the appropriateness of applying research data in primary care. Both groups shared concerns over opportunistic screening, validity, and interpretation of results, increased health system costs and inequities, and genetic discrimination. Primary care providers were ambivalent about the appropriateness and utility of GS in the care of healthy children. Providers feel unprepared and unsure of their obligations in disclosing these results. Providers do not feel they are equipped with the necessary resources and training to support their patients in using GS results in their care.
\end{abstract}

\section{Introduction}

Advances in genomic sequencing (GS) technology, such as genome and exome sequencing, have made it possible to interrogate large amounts of genetic information in a fraction of the time and cost of previous technology. This has facilitated the large-scale use of GS in research and increasingly in clinical settings [1-6]. In research, GS can be used as a tool in studies to understand how genetic variants can contribute to disease development.

Supplementary information The online version of this article (https:// doi.org/10.1038/s41431-019-0547-6) contains supplementary material, which is available to authorized users.

Yvonne Bombard

yvonne.bombard@utoronto.ca

1 Li Ka Shing Knowledge Institute, St. Michael's Hospital, Toronto, ON, Canada

2 University of Toronto, Toronto, ON, Canada

3 The Hospital for Sick Children, Toronto, ON, Canada
Specifically, prospective cohort studies involving healthy children have been used to further scientific knowledge on how genetics can contribute to growth, nutrition, and development [7]. In the clinic, information about an individual's genetic variation can inform treatment, preventative care and diagnosis, particularly in pediatrics. Children may undergo GS to diagnose complex disorders with a probable genetic basis, when other tests, such as single-gene panel testing, have failed to arrive at a diagnosis [8-10].

In either context, GS has the potential to reveal a large amount of detailed information regarding a child's hereditary disposition for other diseases that may be clinically valuable, beyond the information sought after for a clinical indication or research question [11, 12]. Prior literature has indicated that parents are interested in receiving these incidental or secondary findings to inform future planning and care for their children [13, 14]. Parents in these studies often mentioned primarily seeking support and guidance from their primary care providers (henceforth referred to as "providers" for brevity) [13]. Providers will be increasingly tasked with managing these 
secondary findings especially among their healthy pediatric populations involved in GS research. However, the return of secondary findings from GS research amounts to opportunistically screening healthy children for disease risk in the absence of any signs, symptoms, or clinical indication, blurring the lines between research and clinical care $[15,16]$. Primary care providers will be increasingly responsible for not only managing secondary findings from these studies but also informing best practices for conducting pediatric GS research and supporting optimized use of those findings. The objective of this study was to examine provider perspectives towards studies using GS that involve healthy children, evaluate their views on whether or not to provide the results generated by this test, and their preferences for the use of these results in primary care of healthy children.

\section{Methods}

\section{Design}

We conducted qualitative, semistructured interviews between July 2017 and March 2018 with primary care providers involved in the TARGet Kids! Primary Care Practice Based Research Network (PBRN) (www.ta rgetkids.ca). TARGet Kids! is a collaborative research study between healthcare providers and researchers in the departments of Pediatrics and Family and Community Medicine at the University of Toronto, aiming to link early life exposures to disease development and are interested in identifying genetic factors that interfere with growth and development in healthy children aged 0-5. This collaboration is also interested in exploring the use of genome sequencing as a research tool, to obtain diseaserisk information in healthy children as a future extension of the Network's research activities. Ethics approval was obtained from the research ethics boards at both St. Michael's Hospital and The Hospital for Sick Children in Toronto, Ontario.

\section{Participants and recruitment}

Recruitment for this study occurred at three TARGet Kids! affiliated clinics at St. Michael's Hospital. Both family physicians and primary care pediatricians were recruited. Recruitment letters were distributed through email. Participants expressing interest contacted the study coordinator to schedule a telephone interview. Verbal consent was obtained over the phone prior to beginning the interview. We used a purposeful sampling technique to recruit up to 20 providers [17-19].

\section{Data collection}

A semistructured interview guide was used in the audiorecorded telephone interviews of $\sim 30 \mathrm{~min}$ in length, per participant. The guide was informed by the relevant literature and explored providers' clinical experience with genetic testing, and attitudes towards GS and studies involving GS with healthy children (see Appendix A). We explored providers' perceived utility of GS results by probing each individual category of secondary findings that can be returned and asking providers whether they hypothetically should be made available to patients. These categories included: (1) childhood onset medically actionable; (2) childhood onset medically non-actionable; (3) adult-onset medically actionable; (4) adult-onset nonactionable; (5) carrier status. Providers' perceived clinical genetic competencies and their perceived self-efficacy in these competencies were also discussed. Interviews were conducted by CM or SC. Demographic data were collected using questionnaires at the end of each interview.

\section{Data analysis}

Recordings of the telephone interviews were transcribed, anonymized, and qualitatively analyzed through thematic analysis and constant comparison, drawing on grounded theory methodology [17]. The data were first openly coded and labeled using descriptive codes to develop a preliminary codebook. Interviews were coded independently by EJ, CM, and SC. Each code was assessed through discussion and constant comparison among coders to build further iterations of the codebook. Coders reviewed transcripts together to reach consensus on the content of the codes. Coders met regularly to identify and compare codes across various interviews to discern any patterns present in the analysis and to inform possible changes to the interview guide, as is typical in grounded theory [17]. The final step involved axial coding where the interrelationships between codes were identified to develop the underlying theory and emerging phenomenon [19-21].

\section{Results}

A total of 16 interviews were conducted with family physicians or primary care pediatricians, from three TARGet Kids! St. Michael's Hospital sites. Participant demographics are presented in Table 1. Most participants practiced for ten or more years (11/16) and spent ten or more years working with children (10/16).

While most primary care providers saw value in using GS in research for healthy children, opinions diverged on 
Table 1 Primary care provider demographic data.

\begin{tabular}{ll}
\hline Age & $N=16$ \\
\hline$\leq 39$ & 9 \\
$\geq 40$ & 7 \\
Specialty & 11 \\
Family physicians & 5 \\
Pediatricians & \\
Years in clinical practice & 5 \\
$<10$ & 11 \\
$\geq 10$ & \\
Years working with pediatric patients & 6 \\
$<10$ & 10 \\
$\geq 10$ & \\
\hline
\end{tabular}

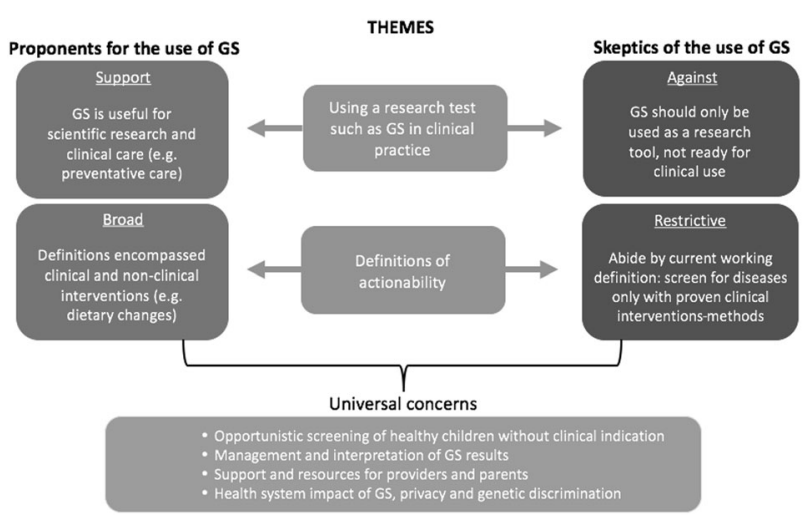

Fig. 1 Summary of primary care provider perspectives on genome sequencing in healthy children and divergence of opinions on two emerging themes between proponents and skeptics.

using the results in primary care for children, resulting in proponents and skeptics. This dichotomy was evident when providers reflected upon their personal experience with genetic testing, such as newborn screening and panel testing in cancer, familiarity with GS and the types of results that can be returned. The differences in opinion were driven by providers' ambivalence about using a research test, such as GS, in clinical care and their uncertainty about what to do with the results (Fig. 1). Proponents of using GS saw value in the results for informing their patients' preventative care and benefiting scientific research as a whole. On the other hand, those who were skeptical about using GS results in primary care were concerned about the technology's reliability, accuracy, and clinical utility. However, proponents and skeptics shared similar concerns around: (1) management of GS results; (2) counseling patients; (3) increased health system costs incurred by this testing; (4) implications of screening without an indication; and (5) the possibility of genetic discrimination. We unpack each of these themes in turn.

\section{Areas of divergence in opinions}

\section{Use of genome sequencing research results in clinical practice}

Providers were ambivalent about using GS results, initially intended for research purposes, in clinical care. Some providers-proponents-supported the use of GS in healthy children for collecting important cohort data for research. They also valued using the information to inform preventative care, such as ordering surveillance tests where indicated by GS results. Examples included monitoring blood-glucose levels or weight, and advising lifestyle changes (e.g., diet and exercise). For example:

"I think it could help tailor the preventative care that they get. It could also provide information about their diet and lifestyle. [If] they see they've got genes for insulin resistance, you may want them to focus around different dietary factors that could help prevent diabetes and fatty liver and things like that down the road."-TKGP01

Interestingly, proponents also acknowledged that the results would be useful for the parents in guiding how they care for their children, and even plan for their future. Some proponents suggested that learning about a child's increased risk for a particular condition might promote preemptive surveillance of symptoms, enhance child-parent bonding and guide developmental support.

Overall, proponents expressed a willingness to learn GS results regardless of whether these results were obtained from a research test because they presumed that the results would be useful to them in tailoring preventative care, surveillance, and support programs.

In contrast, skeptics did not feel that the GS technology was ready for use in clinical care, although they valued its role in advancing genomics research. They disapproved using results obtained from research to guide their patients' management and opportunistically screening their patients without a clinical indication. Others voiced discomfort with using test results that they did not order nor discuss a priori with their patients:

"It's a research study. It's not something that I've clinically arranged, or feel is imperative for their care."-TKGP10

Further, skeptics did not feel they had a professional obligation to use GS results in primary care. They reasoned that unlike commonly ordered laboratory or imaging tests (e.g., blood tests, MRIs, CT-scans), they would not know how to make use of GS results. Therefore, they felt that this 
research data should be kept separate from medical records used in practice. Skeptics also reasoned that GS is "still a research tool, not a tool to be used routinely" (TKPED04) because its utility and "actionability" of results remain questionable.

\section{Definitions of actionability}

The most prevalent theme focused on the utility of GS and the varying ways in which providers conceptualized the actions they can take based on its results. Providers' definitions of what is considered actionable influenced their perspective on which results should be returned to parents from GS, and how they would use these results in primary care. For example, skeptics aligned with traditional, restrictive definitions, and considered results to be actionable only if there are established clinical interventions to reduce morbidity and mortality, whereas proponents had more dynamic definitions of actionability, encompassing nontraditional ideas of possible interventions.

Specifically, proponents diverged from the traditional definition of actionability and typically favored broader definitions, reasoning that there was always some sort of action that can be taken based on GS results, whether it meant advising patients on making lifestyle changes, ordering screening tests, or guiding child rearing.

One of the nonclinical interventions mentioned by proponents was using results to promote lifestyle changes in their patients, such as "more aggressively focusing on their weight, their diet and their exercise" (TKPED04) to prevent or delay the development of at risk diseases.

Proponents also valued returning childhood onset or adult-onset nonactionable results so that parents could proactively improve their children's quality of life and development:

\section{"There are things that could be actionable that could really improve their quality of life. Even things like finding out that they have a learning disability, or dyslexia, it benefits parents to be aware of that, so that they can get the child the support that they need. It's actionable and improves somebody's quality of life."-TKGP01}

In comparison, skeptics employed definitions of actionability that aligned with the traditional definition, restricting what they consider actionable results to situations where there are effective and established clinical interventions for an identified disease risk. Skeptical providers felt that they would struggle with responding to nonactionable results without clear clinical management guidelines. One provider explained the difficulty of knowing a patients' risk for a nonactionable adult-onset disease:
"I wouldn't want to know if they had Huntington's when they're 70. What am I going to do with that as a clinician? Just like I would never order an MRI on a healthy child. Even though I can... what am I going to do with those results?"-TKPED05

Skeptics continuously stressed the importance of returning "clinically useful" results, for instance, returning information about a patient's disease risks would "change what we do, and provides a clear benefit over just knowing" (TKGP13), in addition to possessing a "clear path" (TKGP13) for clinical management. Another provider highlighted the concept of "clinically meaningful," stressing the importance of disease impact in considering which results warranted return (TKGP02).

Skeptics worried about the unintended consequences of returning nonactionable results, such as disrupting parenting and parent-child bonding. While proponents expected GS results to benefit child-parent bonding, skeptics feared returning these results could heighten parents' anxieties and lead to overprotection of their children, resulting in children "not having a normal childhood" (TKGP12) if they perceived their children to be at an increased risk of illness:

"I think anxiety for the child, [parents] and for the family, which could lead to psychosocial interaction issues. It could shelter them from healthy activities that they enjoy. It may set them up to be sort of "bubble children".-_TKPED05

\section{Universal concerns around using GS in healthy children}

Despite the divergence of opinion on the appropriateness and utility of GS results, there were several areas where providers shared common concerns towards the use of GS in healthy children.

Screening healthy children without a clinical indication One area where providers shared concerns was about opportunistically screening healthy children using GS for a broad range of disease risks. They often compared this practice with newborn screening and prenatal screening. Providers discussed traditional criteria for population screening, and pointed out that using GS in healthy children may challenge these established criteria:

"If you do a Pap test. It's screening. It's available to everybody, easy to do, not that expensive. We have good treatments for the outcome. Whereas we don't screen for ovarian cancer right now, because we don't have a good test. We don't have a test that provides us with enough information to make decisions that are 
not going to harm the patient, right. I think that it's still early days and the things that are going to need to be in place are number one, is it available to all?" TKGP01

Providers likened the practice of opportunistically screening healthy children to opening a "Pandora's box" (TKGP11) and offering "blanket screening" (TKPED14) without indication or clear guidelines. They cautioned about being "careful and selective [about] which type of problems we tackle" (TKPED07) because of the inherent complexities of GS (e.g., secondary findings, lack of family history, and risk factors).

Management and interpretation of GS results Many providers felt they lacked the necessary technical expertise and skills to convey GS results to the parents. Some felt unfamiliar with concepts in genetics and expressed discomfort with interpreting and using GS results in managing their patients. Providers were unsure of what obligations they had to their patients in getting in touch with their patients as they became older or as new interpretations of their results came to light. Some feared inducing additional anxiety and/or stress in their patients as a result of their lack of confidence:

"I would be reticent to know what to do with the results. I find it difficult already to get a great handle on genetic results. I find I'm not an expert in that field and I worry I might inadvertently downplay results, or contribute to anxiety by doing searches myself and trying to find helpful information for families." TKPED05

Support and resources for patients and providers Providers were concerned about the additional resources required to facilitate GS testing, pretest and posttest counseling, and additional support or training for themselves. One strategy suggested by the providers was a network of genetic experts (e.g., counselors) to rely on for expertise and help them to "make sense of the disease for the patient" (TKGP02).

Additional recommendations for resources included general guidelines for the return of results and "follow-up plans" (TKGP09). Providers emphasized that without these resources, there could be significant harm done to patients if they were inadequately prepared, educated, and trained to return GS results.

Health system impacts of GS, privacy, and genetic discrimination Providers recognized that these additional resources incurred costs, which could over-burden the healthcare system. One provider worried about "wasting tax payers' dollars" (TKPED05):
"Is it a cost burden to society as a whole? I think we have a responsibility as physicians to try to decipher that and be a gatekeeper, if you will, for technology. "-TKPED05

Others voiced concerns about whether the large volume of genomic data could be stored securely and whether research results should be stored in electronic medical records (EMR). Providers were concerned with researchers possibly having access to these data as apart of patients' EMR, which effectively represents a source of populationwide variant and phenotype information. In particular, they noted the importance of the research team having a concrete plan for the data storage and ensuring its privacy.

Finally, providers feared this testing might render their patients' ineligible for life and/or health insurance and wondered whether they were obligated to disclose their patients' results to insurers or other third parties. Possible genetic discrimination and disease labeling of asymptomatic individuals were also cited as issues to consider in the implementation of GS. See Appendix B for additional illustrative quotes.

\section{Discussion}

This is one of the first studies to report the perspectives of primary care providers on the use of genome sequencing for healthy children. Our results revealed that most providers saw value in using GS in healthy children to answer important research questions; however, opinions diverged on using the results obtained from this research in the clinical care for these children. Proponents valued using secondary findings for informing preventative care whereas skeptics questioned their reliability and clinical utility. Providers also diverged on the "actionability" of GS results; proponents used broader definitions encompassing both clinical and nonclinical interventions, whereas skeptics abided by the traditional definition. Despite this divergence, providers shared similar concerns surrounding opportunistic screening of healthy patients without clinical indication and requiring additional educational support for both patients and providers. Providers also expressed concerns of the cascading costs on the healthcare system, inequities in access, and the possibility of genetic discrimination.

A motivation for using GS results in primary care has been to reduce the cost curve by consolidating diagnostic tests, and informing drug selection/dosing. Yet, there has been little objective information collected on the resource costs in clinical subdisciplines required for adopting GS including: interpretation of results, pretest and post-test counseling, and possible follow-up testing for results [22]. Providers in our study emphasized the need for educational 
training to address their lack of expertise in genomics and interpreting the results. They voiced concerns about the potential economic burden and feasibility issues that would arise, particularly as a result of opportunistic screening, echoing concerns reflected in the literature on the cascades following the return of secondary findings [23, 24].

The continued debate around returning secondary findings in pediatric populations, mostly centered around returning risk information for adult-onset conditions, is further complicated in healthy pediatric populations as there is no manifesting phenotype for which GS is clinically indicated for and all results to become "secondary findings" [25-28]. Therefore, these individuals are effectively being opportunistically screened for disease risk when participating in this kind of research [29]. While some of these screening results could hold clinical utility in preventative care for children, the well-described discrepancy between conceptualizations and definitions of "actionability" between providers that was also observed in our study, make development of necessary guidelines challenging $[25,26]$. Providers often held varying thresholds for actionability with some considering results informing child care for parents to be actionable, reminiscent of arguments supporting reproductive benefit in the newborn screening context [30-34]. Current ACMG guidelines fall short in considering which category of conditions (such as childhood and adult onset) and at what actionable "threshold", require return in either research and clinical settings [35].

Nonetheless, primary care providers and pediatricians are supportive of the return of actionable secondary findings from GS in affected children in the clinical and research contexts [25-28, 36], and in one study, were comfortable making clinical decisions for their adult patients based on the GS results, or seeking out the support of genetics professionals as necessary to make these decisions [37]. However, the widespread disagreement around what is considered to be "in the child's best interest", which is commonly seen in the debate around returning secondary findings for affected children, will likely mean that providers are not any more prepared to return these results for their healthy pediatric patients [38, 39]. Indeed, providers in our study were unsure as to where their obligations lied to patients that seek support for results acquired from a test performed outside of the healthcare system or their immediate circle of care. They acknowledged that these obligations could become long-term and lead to an increased responsibility to maintain contact if a presymptomatic diagnosis is made, disease risk for adult-onset conditions are identified or new interpretations of the results are made available. The expressed unpreparedness of providers from our study highlights a need to further qualitatively assess what is required for providers to be able to effectively manage GS results in terms of education and support, and that their role in managing research results needs to be more clearly defined.

This study had a number of limitations. First, our sample was drawn from outpatient clinics involved in one Primary Care PBRN. The sample included both family physicians and primary care pediatricians in Canada. Further studies might include perspectives of other healthcare providers, such as nurses, social workers, and dietitians, and in diverse practice settings, with direct or indirect experiences with GS, which could contribute to a more comprehensive discussion of using genomic data in clinical care and providers' perceived clinical utility of results.

\section{Conclusion}

Our findings provide novel insight into the perspectives of primary care providers on the use of GS results in the care of healthy children. Secondary findings obtained from GS research could be of clinical relevance to the preventative care of a child. This blurring of the line distinguishing between research and clinical care will drive the need for informed consent procedures to address the concept of unexpected and probabilistic disease risk, and secondary findings with parents of healthy children partaking in GS research. In addition, evidence-based training, education, and support for providers and inclusion of their views in the development of guidelines may be helpful. Future studies to assess the perspectives and preferences of parents of healthy children for using genomic data in their child's healthcare will also further inform these policies.

Acknowledgements We would like to thank the primary care providers that participated in our study for sharing their valuable time and insights with us.

Funding The research presented in this paper was supported by the University of Toronto's McLaughlin Centre. YB was supported by a New Investigator Award from the Canadian Institutes of Health Research (CIHR) during this study.

\section{Compliance with ethical standards}

Conflict of interest The authors declare that they have no conflict of interest.

Publisher's note Springer Nature remains neutral with regard to jurisdictional claims in published maps and institutional affiliations.

\section{References}

1. Grody WW, Thompson BH, Hudgins L. Whole-exome/genome sequencing and genomics. Pediatrics. 2013;132:211-5.

2. Bamshad MJ, Ng SB, Bigham AW, Tabor HK, Emond MJ, Nickerson DA, et al. Exome sequencing as a tool for Mendelian disease gene discovery. Nat Rev Genet. 2011;12:745-55. 
3. Behati S, Tarpey PS. What is next generation sequencing? Arch Dis Child Educ Pr Ed. 2013;98:236-8.

4. Lionel AC, Costain G, Monfared N, Walker S, Reuter MS, Hosseini $\mathrm{SM}$, et al. Improved diagnostic yield compared with targeted gene sequencing panels suggests a role for whole-genome sequencing as a first-tier genetic test. Genet Med. 2018;20:435-43.

5. Pareek CS, Smoczynski R, Tretyn A. Sequencing technologies and genome sequencing. J Appl Genet. 2011;52:413-5.

6. Bombard Y, Bach PB, Offit K. Translating genomics in cancer care. J Natl Compr Canc Netw. 2013;11:1343-53.

7. Golding J. The Avon Longitudinal Study of Parents and Children (ALSPAC) - study design and collaborative opportunities. Eur $\mathbf{J}$ Endocrinol. 2004;151:U119-23.

8. Boycott K, Hartley T, Adam S, Bernier F, Chong K, Fernandez $\mathrm{BA}$, et al. The clinical application of genome-wide sequencing for monogenic diseases in Canada: position statement of the Canadian College of Medical Geneticists. J Med Gen. 2015;52:431-7.

9. Smith HS, Swint JM, Lalani SR, Yamal JM, de Oliveira Otto MC, Castellanos S, et al. Clinical application of genome and exome sequencing as a diagnostic tool for pediatric patients: a scoping review of the literature. Genet Med. 2019;21:3-16.

10. Parsons D, Roy A, Yang Y, Wang T, Scollon S, Bergstrom K, et al. Diagnostic yield of clinical tumor and germline wholeexome sequencing for children with solid tumor. JAMA Oncol. 2016;2:616-24.

11. Burke W, Antommmaria AHM, Bennett R, Botkin J, Clayton EW, Henderson GE, et al. Recommendations for returning genomic incidental findings? We need to talk! Genet Med. 2013;15:854-9.

12. Bombard Y, Robson M, Offit K. Revealing the incidentalome when targeting the tumor genome. J Am Med Assoc. 2013; 310:795-6.

13. Fernandez CV, Bouffet E, Malkin D, Jabado N, O'Connell C, Avard D, et al. Attitudes of parents toward the return of targeted and incidental genomic research findings in children. Genet Med. 2014;16:633-40.

14. Kleiderman E, Knoppers BM, Fernandez CV, Boycott KM, Ouellette G, Wong-Rieger D, et al. Returning incidental findings from genetic research to children: views of parents of children affected by rare diseases. J Med Ethics. 2014;40:691-6.

15. Brothers KB, Vassy JL, Green RC. Reconciling opportunistic and population screening in clinical genomics. Mayo Clin Proc. 2019;94:103-9.

16. Mackley MP, Capps B. Expect the unexpected: screening for secondary findings in clinical genomics research. Brit Med Bull. 2017;122:109-22.

17. Sandelowski M. Sample size in qualitative research. Res Nurs Health. 1995;18:179-83.

18. Morse JM. Designing funded qualitative research. In: Handbook of qualitative research. CA: SAGE Publications Ltd; 1994. p. 220-35.

19. Strauss A, Corbin J. Basics of qualitative research: techniques and procedures for developing grounded theory. CA, USA: SAGE Publications Ltd; 1998.

20. Charmaz KC. Constructing grounded theory: a practical guide through qualitative analysis. London, UK: SAGE Publications Ltd; 2006.

21. Charmaz KC. Qualitative interviewing and grounded theory analysis. In: Holstein JA, Gubrium JF, editors. Inside interviewing: new lenses, new concerns. Thousand Oaks, CA: Sage Publications Inc; 2003. p. 311-30.

22. Foley SB, Rios JJ, Mgbemena VE, Robinson LS, Hampel HL, Toland AE, et al. Use of whole genome sequencing for diagnosis and discovery in the cancer genetics clinic. EBioMedicine. 2015; 2:74-81.

23. Bennette CS, Gallego CJ, Burke W, Jarvik GP, Veenstra DL. The cost-effectiveness of returning incidental findings from nextgeneration genomic sequencing. Genet Med. 2015;17:587-95.

24. Christensen KD, Dukhovny D, Siebert U, Green RC. Assessing the costs and cost-effectiveness of genomic sequencing. J Pers Med. 2015;5:470-86.

25. Lemke AA, Bick D, Dimmock D, Simpson P, Veith R. Perspectives of clinical genetics professionals toward genome sequencing and incidental findings: a survey study. Clin Genet. 2013;84:230-6.

26. Yu J, Harrell TM, Jamal SM, Tabor HK, Bamshad MJ. Attitudes of genetics professionals towards the return of incidental results from exome and whole-genome sequencing. Am J Hum Genet. 2014;95:77-84.

27. Kleiderman E, Avard D, Besso A, Ali-Khan S, Sauvageau G, Hebert J. Disclosure of incidental findings in cancer genomic research: investigators' perceptions on obligations and barriers. Clin Genet. 2015;88:320-6.

28. Knoppers BM, Joly Y, Jacques S, Durocher F. The emergence of an ethical duty to disclose genetic research results: international perspectives. Eur J Hum Genet. 2006;14:1170-8.

29. Lindor NM, Thibodeau SN, Burke W. Whole-genome sequencing in healthy. People Mayo Clin Proc. 2017;92:159-72.

30. Bombard Y, Miller FA, Barg CJ, Patton SJ, et al. A secondary benefit: the reproductive impact of carrier results from newborn screening for cystic fibrosis. Genet Med. 2017;19:403-11.

31. Bombard Y, Miller FA, Hayeems RZ, Wilson BJ, et al. Healthcare providers' views on pursuing reproductive benefit through newborn screening: the case of sickle cell disorders. Eur J Hum Genet. 2012;20:498-504.

32. Bombard Y, Miller A, Hayeems RZ, Avard D, Knoppers BM. Reconsidering reproductive benefit through newborn screening: a systemtic review of guidelines on preconception, prenatal and newborn screening. Eur J Hum Genet. 2010;18:751-60.

33. Bombard Y, Miller FA, Hayeems RZ, Avard D, Knoppers BM, Cornel MC, et al. The expansion of newborn screening: is reproductive benefit an appropriate pursuit? Nat Rev Genet. 2009; 10:666-7.

34. Pereira S, Robinson JO, Gutierrez AM, Petersen DK, Hsu RL, Lee $\mathrm{Ch}$, et al. Perceived benefits, risks and utility of newborn genomic sequencing in the BabySeq project. Pediatrics. 2019;143:S6-S13.

35. Green RC, Berg JS, Grody WW, Kalia SS, Korf BR, Martin CL, et al. ACMG recommendations for reporting of incidental findings in clinical exome and genome sequencing. Genet Med. 2013; 15:565-74.

36. Ormondroyd E, Mackley MP, Blair E, Craft J, Knight JC, Taylor JC, et al. "Not pathogenic until proven otherwise": perspectives of UK clinical genomics professionals toward secondary findings in context of a Genomic Medicine Multidisiplinary Team and the 100,000 genomes project. Genet Med. 2018;20:320-8.

37. Vassy JL, Christensen KD, Schonman EF, Blout CL, Robinson JO, Krier JB, et al. The impact of whole-genome sequencing on the primary care and outcomes of healthy adult patients: a pilot randomized trial. Ann Intern Med. 2017;167:159-69.

38. Delanne J, Nambot S, Chassagne A, Putois O, Pelissier A, Peyron $\mathrm{C}$, et al. Secondary findings from whole-exome/genome sequencing evaluating stakeholder perspectives. A review of the literature. Eur J Med Genet. 2019;62:103529.

39. Strong KA, Zusevics KL, Bick D, Veith R. Views of primary care providers regarding the return of genome sequencing incidental findings. Clin Genet. 2014;8:461-8. 